\title{
Abundancia estacional de crustáceos asociados a la captura artesanal de jaiba azul (Callinectes sapidus) en Sisal, Yucatán, México
}

\section{Seasonal abundance of crustaceans associated with artisanal fishery of blue crab (Callinectes sapidus) in Sisal, Yucatan, Mexico}

\author{
José Alfredo Celis-Sánchez ${ }^{*}$, Arely de Jesús Estrella-Canto ${ }^{1}$, Gaspar Román Poot-López ${ }^{1}$, \\ Carlos González-Salas ${ }^{1}$ y Jorge Alberto López-Rocha ${ }^{2}$
}

\begin{abstract}
RESUMEN
Se estudiaron la abundancia estacional y la selectividad de las principales especies de crustáceos asociadas a la pesca de la jaiba azul, en el puerto de Sisal, Yucatán, México. Para ello se utilizaron 52 trampas jaiberas, divididas en cuatro transectos colocados de manera paralela, a una distancia de 100, 150, 200 y $250 \mathrm{~m}$ de la línea de costa. Cada transecto estuvo conformado por 13 trampas, con una distancia de $20 \mathrm{~m}$ una de otra. Las trampas se colocaron al atardecer y se revisaron al amanecer, estandarizando el esfuerzo de pesca en 17 horas/trampa/día. En total se capturaron 832 organismos de ocho especies, sin embargo, el estudio se realizó con cuatro especies de importancia comercial: jaiba azul (Callinectes sapidus), jaiba blanca (C. similis), cangrejo araña (Libinia dubia) y cangrejo moro (Menippe mercenaria), representando el 60.7, 13.3, 11.4 y $11.6 \%$ de las capturas, respectivamente. Al dividir la captura total entre épocas climáticas (secas, lluvias y nortes), se detectaron diferencias significativas en la captura por unidad de esfuerzo (kg/trampa). C. sapidus y L. dubia registraron su máxima captura en la época de secas, sin embargo, C. similis y M. mercenaria la presentaron en la época de nortes. La mayoría de las especies presentaron una proporción sexual diferente a una relación 1:1, con excepción de $C$. similis. Con respecto a la selectividad o talla de primera captura $\left(\mathrm{L}_{50}\right)$, los valores fueron de 148, 90, 60 y 94 mm de ancho de caparazón, para C. sapidus, C. similis, L. dubia y M. mercenaria, respectivamente.
\end{abstract}

Palabras claves: Callinectes, Libinia, Menippe, selectividad, CPUE.

\begin{abstract}
Seasonal abundance and selectivity of the main crustacean species associated with blue crab fishing was studied at the port of Sisal, Yucatan, Mexico. For this purpose, $52 \mathrm{crab}$ traps were used, which were divided into four parallel transects placed 100, 150, 200 and $250 \mathrm{~m}$ of the shoreline. Each transect consisted of 13 traps $20 \mathrm{~m}$ from each other. Traps were set at dusk and checked at dawn, standardizing the fishing effort to 17 hours/trap/day. A total of 832 organisms from eight species were caught; however, the study was conducted with four commercially important species: Blue Crab (Callinectes sapidus), white crab (C. similis), spider crab (Libinia dubia) and stone crab (Menippe mercenaria), accounting for $60.7 \%$, $13.3 \%, 11.4 \%$, and $11.6 \%$ of the catch, respectively. When dividing the total catch between climatic seasons (dry, rainy, and north wind), significant differences were detected in catch per unit effort ( $\mathrm{kg}$ trap). The highest catch of C. sapidus and L. dubia was recorded in the dry season, while C. similis and M. mercenaria highest catch was in the north wind season. Most species showed a sex ratio different from 1:1, except for $C$. similis. Regarding selectivity or size at first catch $\left(\mathrm{L}_{50}\right)$ values were 148, 90, 60, and $94 \mathrm{~mm}$ carapace width for C. sapidus, C. similis, L. dubia and M. mercenaria, respectively.
\end{abstract}

Keywords: Callinectes, Libinia, Menippe, selectivity, CPUE.

I Universidad Autónoma de Yucatán. Campus de Ciencias Biológicas y Agropecuarias. Carretera Mérida-Xmatkuil Km. 15.5, C.P.: 97315, Mérida, Yucatán, México. alfredo.celis87@hotmail.com*

2 Unidad Multidisciplinaria de Docencia e Investigación, Facultad de Ciencias de la UNAM, México-Yucatán-SisalPuerto de abrigo.

Recibido: 30 de marzo de 2014

Corregido: 4 de junio de 2014

Aceptado: 8 de julio de 2014

DOI: http://dx.doi.org/10.15359/revmar.6.5 


\section{INTRODUCCIÓN}

Mundialmente, las pesquerías de crustáceos son de gran importancia en diferentes regiones costeras, en especial las langostas, los cangrejos y camarones, debido a que presentan una alta demanda en el mercado internacional (Tully et al. 2003). Los crustáceos decápodos son recursos que pueden soportar pesquerías comerciales y domésticas, por su valor económico, además de que presentan un papel primordial en la cadena trófica, ya que son presa para diversas especies $\mathrm{y}$, a su vez, son voraces depredadores de otras (Moreno et al. 2011). En México, la pesquería de jaiba es una actividad realizada tanto en el litoral del Pacífico como en el Golfo de México y en el mar Caribe. Sin embargo, esta pesquería presenta como fauna de acompañamiento a otras especies de crustáceos, principalmente de las familias Menippidae y Majidae, que no son descartadas debido a su importancia comercial (Mexicano-Cíntora et al. 2007; Magaña-Gallegos et al. 2012).

En el año 2004, en el Estado de Yucatán, México, la jaiba azul $(C$. sapidus) presentó una captura anual de $71.53 \mathrm{t}$, con un precio de $\$ 14.66$ pesos mexicanos por $\mathrm{kg}$. Los principales puertos donde este recurso es capturado son: Celestún (55\%), Dzilam (10\%) y Telchac Puerto (8\%). En tanto, para el maxquil o cangrejo araña (L. dubia) se han reportado capturas anuales de $8.46 \mathrm{t}$ y con un precio de $\$ 8$ por kg; el puerto principal de captura es Celestún, Yucatán, y se realiza durante los meses de mayo a agosto (Mexicano-Cíntora et al. 2007). Para el cangrejo moro
(Menippe mercenaria), el precio se ha establecido en US\$8.22 (MagañaGallegos et al. 2012). No obstante, $M$. mercenaria y $L$. dubia son destinados principalmente para su uso como carnada para la pesca de pulpo (Octopus maya y $O$. vulgaris), práctica muy arraigada en dicho Estado (Gavaldón \& Fraga, 2004; Mexicano-Cíntora et al. 2007). La comercialización de jaiba generalmente se desarrolla en restaurantes locales, representando una fuente importante de ingresos para las familias de los pescadores (UcEspadas, 2007).

Con el fin de establecer estrategias de ordenación preventiva para las pesquerías, es importante obtener información básica de las especies, las técnicas de captura, la captura por unidad de esfuerzo (CPUE) y la selectividad de las artes de pesca, ya que pueden proporcionar información suficiente para la gestión de la pesquería (Perry et al. 1999; Smith \& Addison, 2003). En Sisal, Yucatán, la pesquería de crustáceos decápodos es una actividad reciente, por lo tanto, no presenta medidas de regulación, lo cual la hace susceptible de ser explotada todo el año. De acuerdo con el Diario Oficial de la Federación, la talla mínima de captura establecida en México para las jaibas es de $110 \mathrm{~mm}$ de ancho de caparazón (DOF, 1974), sin embargo, esta medida se estable para diferentes especies de jaiba. En este sentido, el presente trabajo pretende contribuir al conocimiento de la pesquería de jaiba en la región y aportar información biológica de las especies de crustáceos asociadas a dicha actividad. 


\section{MATERIALES Y MÉTODOS}

Área de estudio: Sisal es un puerto que forma parte del municipio de Hunucmá. Este se encuentra ubicado al poniente del Estado de Yucatán, en el Golfo de México y está conformado por 1837 habitantes (Fig. 1). Se ubica en los $21^{\circ} 09^{\prime} 55^{\prime \prime} \mathrm{N}$ y $90^{\circ} 01^{\prime} 50^{\prime \prime} \mathrm{O}$.

El poblado se encuentra a 53 $\mathrm{km}$ al noroeste de Mérida, Yucatán (INEGI, 2010). Sisal posee un clima cálido húmedo, con una temperatura que oscila entre $27^{\circ} \mathrm{C}$, con una precipitación diaria de $0.2 \mathrm{~mm}$, presenta un porcentaje de humedad del $81 \%$ y una presión de $1013 \mathrm{mb}$. Inciden en esta zona vientos del norte y ciclones tropicales. Asimismo, debido a su localización en la zona tropical está influenciada por tres períodos climáticos: secas (marzojunio), lluvias (julio-octubre) y nortes (noviembre-febrero), que determinan la dinámica ambiental y ecológica del sistema (Vega-Cendejas, 2004).

Técnica de muestreo: Se definió una zona de muestreo en la costa de Sisal, Yucatán, México, de acuerdo con las recomendaciones de pescadores locales. Durante los meses de febrero a diciembre de 2013, se realizaron seis salidas de pesca cada dos meses (febrero, abril, junio, agosto, octubre y diciembre). En cada salida de pesca se utilizaron 52 trampas jaiberas de $50 \times 30 \times 30 \mathrm{~cm}$, con una luz de malla de $2 \mathrm{~cm}$ y $12 \mathrm{~cm}$ de diá-

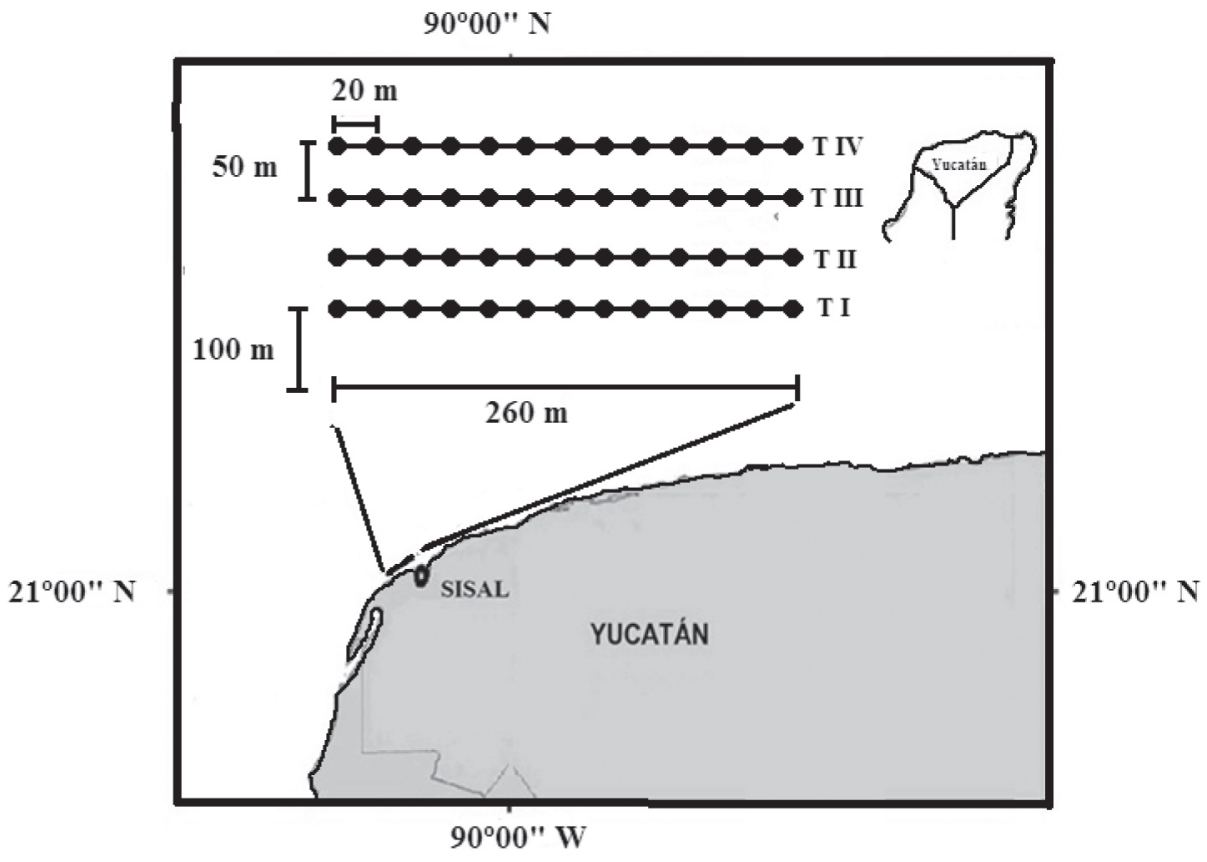

Fig. 1. Ubicación de los cuatro transectos en el puerto de Sisal, Yucatán, México. TI $($ transecto I) $=1.48 \mathrm{~m}$, TII (transecto II) $=1.96 \mathrm{~m}$, TIII (transecto III) $=3.0 \mathrm{~m}$, TIV (transecto IV $=3.5 \mathrm{~m}$ )

Fig.1. Location of the four transects in the port of Sisal, Yucatan, Mexico. TI (transect I) $=1.48 \mathrm{~m}$, TII $($ transect II $)=1.96 \mathrm{~m}$, TIII $($ transect III $)=3.0 \mathrm{~m}$, TIV $($ transect IV $)=3.5 \mathrm{~m}$ 
metro de boca de entrada, similares a las que utilizan los pescadores de manera comercial. Las trampas se dividieron en cuatro transectos (13 trampas/transecto) que fueron ubicados de manera paralela a la costa. El transecto I se ubicó a 100 $\mathrm{m}$ de la orilla y los transectos II, III y IV fueron colocados a 150, 200 y 250 de la línea de costa, respectivamente. Las trampas de un mismo transecto se colocaron a una distancia de $20 \mathrm{~m}$ una de otra, formando una línea recta de $260 \mathrm{~m}$. Para arrojar las trampas en cada zona se utilizó una embarcación ( 25 pies de eslora) con motor fuera de borda (40 hp). Las trampas se colocaron al atardecer y se revisaron al amanecer, estandarizando el esfuerzo de pesca a 17 horas/trampa. La carnada consistió en restos de pescado (subproductos del fileteo) de bajo valor comercial (300 g por trampa). Las artes, métodos de captura y esfuerzo de pesca se diseñaron de acuerdo con las técnicas tradicionales de captura que emplean los pescadores locales. Con el fin de obtener parámetros fisicoquímicos del agua del puerto de Sisal, se llevaron a cabo los siguientes registros: en cada jornada de pesca se midió la salinidad y temperatura del agua con una sonda marca YSI Pro 2030, la profundidad se midieron con una sondaleza marcada a intervalos de $10 \mathrm{~cm}$.

Análisis de las capturas: Una vez que las trampas fueron extraídas del agua, los organismos capturados se identificaron por especie, según la clasificación de Raz-Guzmán et al. (1992). Se midió el ancho del caparazón (AC), tomando la longitud entre las bases de las espinas más distantes del caparazón, para ello se utilizó un vernier/regla con precisión de $0.01 \mathrm{~mm}$, y se les tomó el peso en gramos con una balanza granataria de una precisión de $0.01 \mathrm{~g}$. Se estimó la captura por unidad de esfuerzo (CPUE) dividiendo el peso total de los organismos (por especie) capturados por trampa al día.

Para determinar si existen diferencias significativas en la CPUE entre especies y épocas climáticas se aplicó un ANOVA de dos vías. Al igual que la CPUE entre la profundidad y las especies. No obstante, debido a que los datos de la CPUE no cumplieron con el supuesto de normalidad, los datos fueron transformados con el método de Box-Cox para cumplir dichos supuestos (Sakia, 1992) y se aplicaron las pruebas posteriores de comparaciones múltiples de Turkey HSD. Asimismo, debido a que los datos de la CPUE total (la cuatro especies) no cumplieron con el supuesto de normalidad, se procedió a realizar una prueba no paramétrica Kruskal-Wallis para comparar dicho parámetro (CPUE) entre épocas climáticas (secas, lluvias y nortes).

Proporción de sexos: $\mathrm{La}$ proporción macho: hembra se determinó dividiendo el número de machos entre hembras. Con la finalidad de establecer si la proporción de machos y hembras difiere o no significativamente de una proporción $1: 1$, se aplicó una prueba de bondad de ajuste, usando el estadístico de chicuadrada $\left(X^{2}\right)$ (Moreno et al. 2011).

Relación ancho del caparazónpeso: Se determinó la relación AC-Peso, 
la cual describió una curva que relaciona el ancho del caparazón (AC) con el peso $(P)$ de la jaiba y demás crustáceos de acuerdo con la siguiente ecuación:

$$
P_{t}=a(\mathrm{AC})^{b}
$$

Los parámetros $a$ (intercepto) y $b$ (pendiente) se estimaron por regresión lineal basada en los logaritmos de AC y el peso de acuerdo con:

$$
\log P=\log a+b \log A C
$$

El parámetro $b$ determina si la proporción del peso con respecto a la longitud del caparazón mantiene una proporción al cubo $(b=3$, crecimiento de tipo isométrico) o difiere de esta $(b \neq 3$, alométrico). Para determinar el tipo de crecimiento de las especies de crustáceos se aplicó una prueba t-student para probar si $b \neq 3$ (Velázquez-Abunader et al. 2012).

Selectividad del arte de pesca: Con los datos de AC de las especies capturadas se realizaron histogramas de frecuencias de tallas con intervalos de clase de $10 \mathrm{~mm}$, con el fin de observar con mayor detalle los diferentes grupos presentes en una distribución. El histograma de frecuencias de las longitudes de AC se utilizó para ajustar los parámetros de la ecuación de selectividad, con base en la ecuación:

$$
r(\mathrm{AC})=\frac{\exp \left(b_{0}+b_{1} \mathrm{AC}\right)}{1+\exp \left(b_{0}+b_{1} \mathrm{AC}\right)}
$$

Donde $r(A C)$ es la probabilidad de retención de un $\mathrm{AC}$ determinado, $b_{0}$ y $b_{1}$ son constantes. La ecuación se ajustó mediante mínimos cuadrados utilizando la función Solver de Microsoft Excel, dicha función minimiza el error cuadrático entre los valores observados y simulados. Se estimaron los siguientes parámetros: (i) $\mathrm{L}_{25}, \mathrm{~L}_{50}$ y $\mathrm{L}_{75}$, que representan respectivamente el $\mathrm{AC}$ a los cuales el $25 \%, 50 \%$ y el $75 \%$ de los crustáceos son retenidos por la trampa; (ii) el rango de selección (RS), como la diferencia entre $\mathrm{L}_{75}$ y L $\mathrm{L}_{25}$ (Millar \& Fryer, 1999; Arellano-Torres et al. 2006).

$L_{30}=-\frac{b_{0}}{b_{1}} ; R S_{50}=-\frac{2 \log _{\mathrm{e}}(3)}{b_{1}} ; L_{25}=L_{50}-\frac{R S}{2} ; L_{75}=L_{50}+\frac{R S}{2}$.

\section{RESULTADOS}

Durante el periodo de febrero a diciembre de 2013, en el puerto de Sisal, Yucatán, México, se capturaron un total de 832 crustáceos decápodos pertenecientes a ocho especies (dos especies solo se identificaron por género y seis por especie). Las tres familias identificadas fueron: Portunidae (Callinectes sapidus, C. similis, Arenaeus cribarius y Portunus spinimanus), Majidae ( $L$. dubia y Libinia sp.) y Menippidae (M. mercenaria y Calappa sp.). En términos de captura, las especies que presentaron mayor biomasa fueron: la jaiba azul (C. sapidus) con un $60.7 \%$, la jaiba blanca (C. similis) con un $13.3 \%$, el maxquil o cangrejo araña (L. dubia) con un $11.4 \%$ y el cangrejo moro $(M$. mercenaria) con un $11.6 \%$, obteniendo una biomasa total de $106.11 \mathrm{~kg}$ (Fig. 2). No obstante, para la evaluación de parámetros poblacionales y pesqueros 


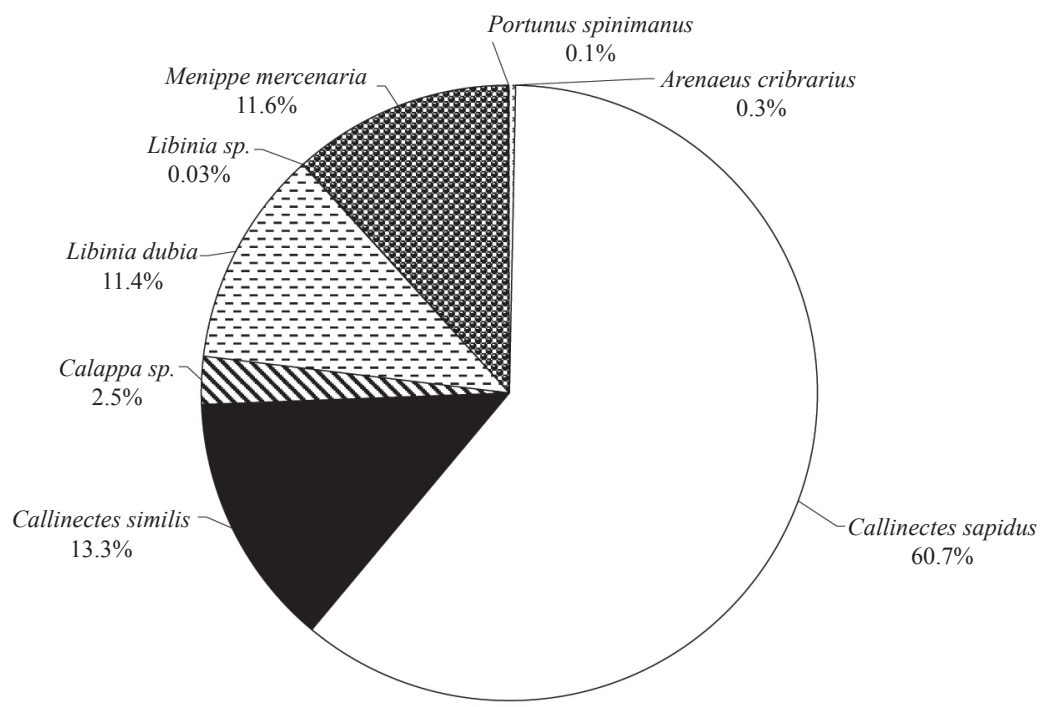

Fig. 2. Biomasa relativa de las especies de crustáceos decápodos asociadas a la captura de jaiba azul (Callinectes sapidus) en Sisal, Yucatán, México

Fig. 2. Relative biomass of the main decapod crustacean species associated with the fishery of blue crab (Callinectes sapidus) in Sisal, Yucatan, Mexico

fueron descartados los individuos de las especies Calappa sp., A. cribarius, P. spinimanus y Libinia $\mathrm{sp}$. debido a su baja abundancia y nula importancia económica en la zona.

Los parámetros fisicoquímicos se muestran en el cuadro 1, donde se muestran las variables de temperatura y salinidad a lo largo del periodo de estudio. Debido a la ubicación de los transectos (I, II, III y IV) de manera paralela a la costa, se presentó un incremento en la profundidad del agua a medida que los transectos se colocaron a mayor distancia de esta. La profundidad para el transecto I fue de $1.48 \pm 0.36 \mathrm{~m}$, el transecto II registró una profundidad de $1.96 \pm 0.36 \mathrm{~m}$, el transecto III presentó un promedio de $3.0 \pm 0.33 \mathrm{~m}$ de profundidad y el transecto IV tuvo un promedio de 3.51 $\pm 0.25 \mathrm{~m}$ de profundidad.

\section{Captura por unidad de esfuerzo} (CPUE): De las cuatro especies consideradas en el estudio, C. sapidus presentó la mayor abundancia en las tres épocas climáticas (Fig. 3). En la época de secas, $L$. dubia y $C$. sapidus registraron su máxima CPUE. Por otra parte, $M$. mercenaria y $C$. similis exhibieron su máxima CPUE en la temporada de nortes. Con respecto a $L$. dubia, no se capturaron individuos en la época de nortes. Al comparar la CPUE entre especies y épocas climáticas, el ANOVA de dos vías no detectó diferencias significativas entre épocas climáticas $\left(\mathrm{F}_{0.05,3,2}=0.103\right)$, pero sí entre especies $\left(\mathrm{F}={ }_{0.05,3,2}=0.001\right)$. C. sapidus registró diferencias significativas con $C$. similis, M. mercenaria y L. dubia.

En la figura 4 se muestran las CPUE de las especies asociadas a la pesca de jaiba, por transecto. $C$. sapidus exhibió una CPUE constante en los cuatro transectos, sin embargo, 
Cuadro 1. Temperatura y salinidad del agua en Sisal, Yucatán, México, por temporada climática Table 1. Water temperature and salinity in Sisal, Yucatan, Mexico by climatic season

\begin{tabular}{ccc}
\hline Época climática & Temperatura $\left({ }^{\circ} \mathbf{C}\right)$ & Salinidad (UPS) \\
\hline Nortes & $25.0 \pm 1.06$ & $31.98 \pm 1.09$ \\
Secas & $29.30 \pm 1.13$ & $33.63 \pm 0.60$ \\
Lluvias & $29.90 \pm 1.06$ & $31.11 \pm 0.86$ \\
\hline
\end{tabular}

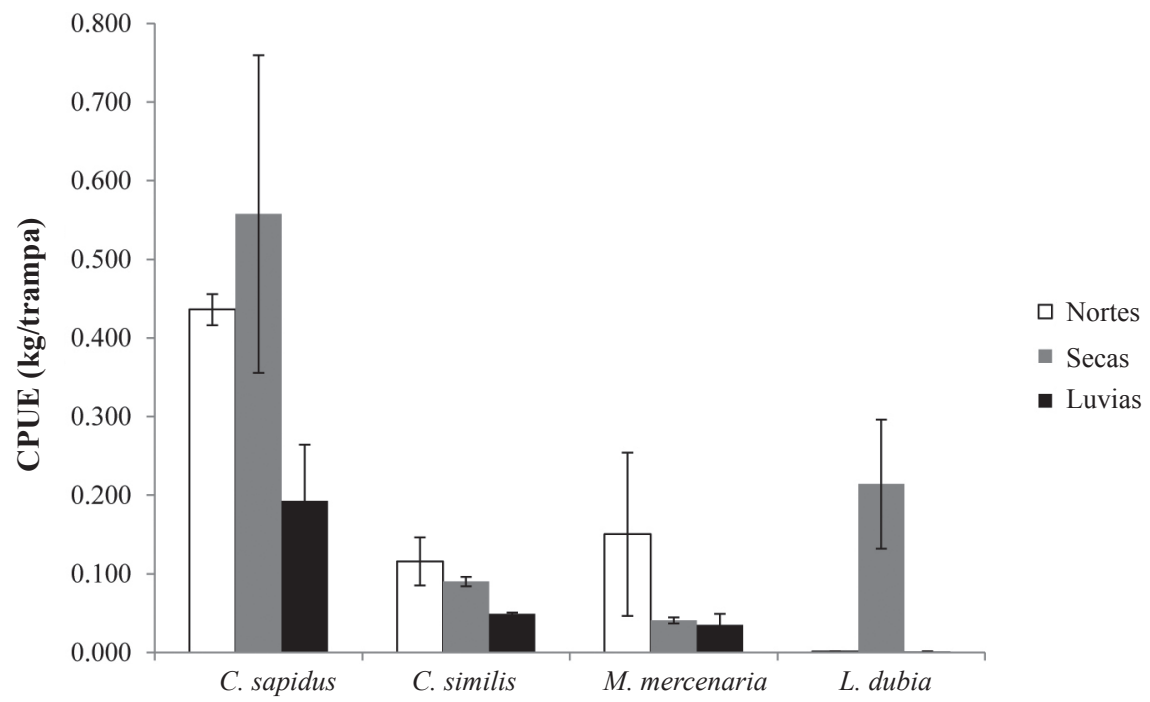

Fig. 3. CPUE (kg/trampa) de las principales especies de crustáceos asociadas a la pesca de jaiba azul en tres épocas climáticas

Fig. 3. CPUE (kg/trap) of the main crustacean species associated with blue crab fishing in the three climatic seasons

esta especie presentó su máxima CPUE en el transecto II. C. similis presentó su máxima CPUE en los transectos I y IV. Por otra parte, M. mercenaria registró su mayor captura en el transecto III, exhibiendo la mínima CPUE para el transecto I. Con respecto a L. dubia, presentó una CPUE baja para el transecto I, incrementándose considerablemente en el transecto II, con respecto a las otras especies. Al comparar la CPUE entre especies y profundidad, el ANOVA de dos vías no detectó diferencias significativas entre la profundidad $\left(\mathrm{F}={ }_{0.005,3,3}=0.157\right)$, pero sí entre especies $\left(\mathrm{F}={ }_{0.005,3,3}=0.000\right)$. Por otra parte, $C$. sapidus y $C$. similis presentaron diferencias significativas con M. mercenaria y L. dubia.

Con respecto a la comparación de la CPUE registrada entre las temporadas climáticas, la temporada de secas presentó mayor CPUE con $0.21 \mathrm{~kg} /$ trampa, seguido de la temporada de nortes con $0.17 \mathrm{~kg} /$ trampa $\mathrm{y}$, por último, la temporada de lluvias con $0.06 \mathrm{~kg} /$ trampa. Ahora bien, al comparar la CPUE con las diferentes épocas climáticas, el 


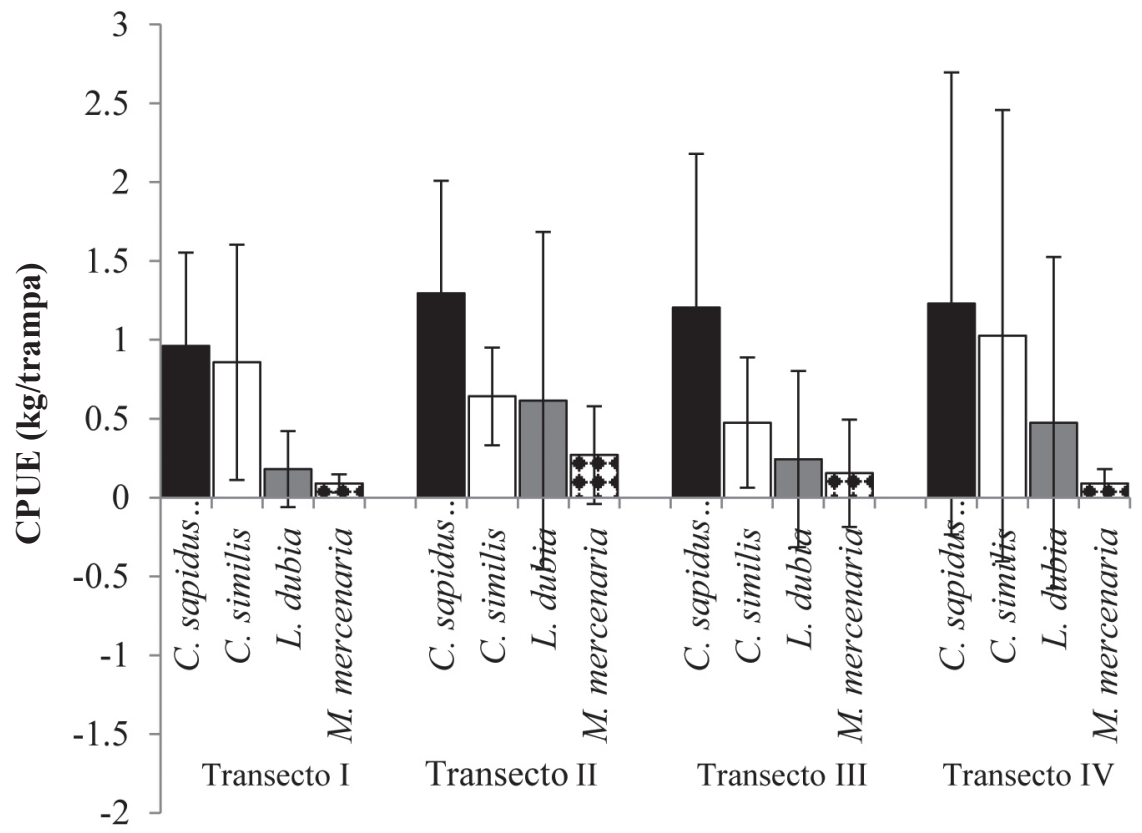

Fig. 4. CPUE (kg/trampa) de las principales especies de crustáceos asociadas a la pesca de jaiba por transecto (diferentes profundidades)

Fig. 4. CPUE (kg/trap) of the main crustacean species associated with the blue crab fishing by transect (different depths)

estadístico de Kruskal-Wallis indicó que no se encontraron diferencias significativas $\left(\mathrm{F}_{0.05,3,2}=0.4265\right)$.

Proporción de sexos: Se obtuvo un total de 354 individuos de C. sapidus, de los cuales 61 fueron machos y 293 hembras, lo que equivale a una proporción de 1:4.8 machos: hembras (M:H), presentando diferencias significativas a una proporción 1:1 $(P<0.05)$, lo mismo sucedió para L. dubia, ya que de los 128 individuos recolectados 90 fueron hembras y 38 fueron machos, equivalente a una proporción de 1:2.4 M:H. Por otra parte, C. similis exhibió una captura total de 236 especímenes, de los cuales 118 fueron machos y 118 fueron hembras, por lo tanto, $C$. similis no presentó diferencias significativas con la relación 1:1 $(P>0.05)$. Finalmente, $M$. mercenaria registró un total de 44 individuos, de los cuales 34 fueron hembras y 10 machos, equivalente a una proporción sexual de 1:3.4 M:H, por lo tanto, la población de $M$. mercenaria presentó diferencias significativas $(P<0.05)$ con respecto a $1: 1$.

Relación ancho del caparazón (AC)-Peso (P): Los valores de los parámetros de la ecuación AC-Peso se presentan en el cuadro 2, donde se señalan aquellas especies con crecimiento isométrico y alométrico, además de los máximos y mínimos de los pesos y el AC de los organismos capturados.

Talla de primera captura: Con respecto a la talla de primera captura $\left(\mathrm{L}_{50}\right)$ 
Cuadro 2. Valores constantes de la relación ancho del caparazón-peso y tipo de crecimiento para cada una de las principales especies de crustáceos decápodos capturadas en el puerto de Sisal, Yucatán, México

Table 2. Constant values of the shell width/weight and type of growth ratio for each of the main species of decapod crustaceans caught in the port of Sisal, Yucatan, Mexico

\begin{tabular}{|c|c|c|c|c|c|c|}
\hline Especie & Sexo & $\mathrm{AC}(\mathrm{mm})$ & Peso (g) & Parámetros & T-student & Crecimiento \\
\hline \multirow{3}{*}{ C. sapidus } & A. sexos & $82-188$ & $45-394.3$ & $\mathrm{P}=0.0014 \mathrm{AC}^{2.3397}$ & $t=8.89, P>0.05$ & $\begin{array}{l}\text { Alométrico } \\
(\beta \neq 3)\end{array}$ \\
\hline & Hembras & $86-188$ & $523-316.5$ & $\mathrm{P}=0.0025 \mathrm{AC}^{2.221}$ & $t=969, P>0.05$ & $\begin{array}{c}\text { Alométrico } \\
(\beta \neq 3)\end{array}$ \\
\hline & Machos & $82-188$ & $45-394.3$ & $\mathrm{P}=0.0002 \mathrm{AC}^{2.8323}$ & $t=1.32, P<0.05$ & $\begin{array}{l}\text { Isométrico } \\
\qquad(\beta=3)\end{array}$ \\
\hline \multirow{3}{*}{ C. similis } & A. sexos & $125-160$ & $12.5-145$ & $\mathrm{P}=0.0006 \mathrm{AC}^{2.5333}$ & $t=3.96, P>0.05$ & $\begin{array}{l}\text { Alométrico } \\
\qquad(\beta \neq 3)\end{array}$ \\
\hline & Hembras & $64-110$ & $14-94.2$ & $\mathrm{P}=0.00003 \mathrm{AC}^{3.17}$ & $t=1.20, P<0.05$ & $\begin{array}{l}\text { Isométrico } \\
(\beta=3)\end{array}$ \\
\hline & Machos & $44-160$ & $12.5-145$ & $\mathrm{P}=0.0033 \mathrm{AC}^{2.1557}$ & $t=4.79, P>0.05$ & $\begin{array}{l}\text { Alométrico } \\
\qquad(\beta \neq 3)\end{array}$ \\
\hline \multirow{3}{*}{ L. dubia } & A. sexos & $45-110$ & $24-250$ & $\mathrm{P}=0.0562 \mathrm{AC}^{1.8021}$ & $t=7.16, P>0.05$ & $\begin{array}{l}\text { Alométrico } \\
\qquad(\beta \neq 3)\end{array}$ \\
\hline & Hembras & $50-108$ & $42-176$ & $\mathrm{P}=0.3396 \mathrm{AC}^{1.3537}$ & $t=8.18, P>0.05$ & $\begin{array}{l}\text { Alométrico } \\
\qquad(\beta \neq 3)\end{array}$ \\
\hline & Machos & $45-110$ & $24-250$ & $\mathrm{P}=0.0117 \mathrm{AC}^{2.2066}$ & $t=2.96, P>0.05$ & $\begin{array}{l}\text { Alométrico } \\
\qquad(\beta \neq 3)\end{array}$ \\
\hline \multirow{3}{*}{$\begin{array}{c}M . \\
\text { mercenaria }\end{array}$} & A. sexos & $56-110$ & $60.2-697$ & $\mathrm{P}=0.0005 \mathrm{AC}^{2.8819}$ & $t=8.89, P>0.05$ & $\begin{array}{l}\text { Isométrico } \\
\quad(\beta=3)\end{array}$ \\
\hline & Hembras & $56-120$ & 67.1-684 & $\mathrm{P}=0.0013 \mathrm{AC}^{2.6642}$ & $t=8.89, P>0.05$ & $\begin{array}{l}\text { Isometrico } \\
\qquad \beta=3)\end{array}$ \\
\hline & Machos & $68-140$ & $60.2-697$ & $\mathrm{P}=0.00008 \mathrm{AC}^{3.2957}$ & $t=8.89, P>0.05$ & $\begin{array}{l}\text { Isométrico } \\
\qquad \beta=3)\end{array}$ \\
\hline
\end{tabular}

de los crustáceos capturados, C. sapidus presentó la mayor $\mathrm{L}_{50}$ con $147.90 \mathrm{~mm}$ de AC (Fig. 5a), en tanto los valores de $\mathrm{L}_{25}$ $\mathrm{y} \mathrm{L}_{75}$ fueron de 137.91 y $157.88 \mathrm{~mm}$ de $\mathrm{AC}$, respectivamente. Para $C$. similis, la $\mathrm{L}_{50}$ fue de $90.17 \mathrm{~mm}$ de $\mathrm{AC}$ (Fig. 5b), y la $\mathrm{L}_{25} \quad \mathrm{y} \mathrm{L}_{75}$ se establecieron en 84.94 y $96.06 \mathrm{~mm}$ de $\mathrm{AC}$, respectivamente. Para L. dubia, la $\mathrm{L}_{50}$ se estableció en $60.25 \mathrm{~mm}$ de $\mathrm{AC}$ (Fig. 5c), con una $\mathrm{L}_{25}$ y $\mathrm{L}_{75}=$ de 55.95 y $64.55 \mathrm{~mm}$ de $\mathrm{AC}$, respectivamente. Para $M$. mercenaria la $\mathrm{L}_{50}$ fue de $93.82 \mathrm{~mm}$ de $\mathrm{AC}$ (Fig. 5d), con una $\mathrm{L}_{25}$ y $\mathrm{L}_{75}=$ de 80.10 y 107.53 $\mathrm{mm}$ de AC, respectivamente. En el cuadro 3 se muestran los valores de máxima verosimilitud de los parámetros de las curvas de selectividad.

\section{DISCUSIÓN}

En el presente estudio, la jaiba azul (C. sapidus) representó el $60.7 \%$ de la captura total, colocándola como la especie dominante en la costa de Sisal, Yucatán, seguida por C. similis, $M$. mercenaria $\mathrm{y}$, por último, $L$. dubia. Lo anterior se debe a la mayor movilidad que tienen las jaibas $(C$. sapidus y $C$. similis) para localizar alimento y ocupar diversos hábitats y profundidades (Fitz \& Wiegert, 1992; Ortiz-León et al. 2007), ya que el área de acción de las trampas para la pesca de crustáceos puede ser de 13.4-30 m a la redonda de cada trampa (Ahumada \& Arana, 2009). En Santiago de Cuba, Gómez-Luna et al. (2009) llevaron a 

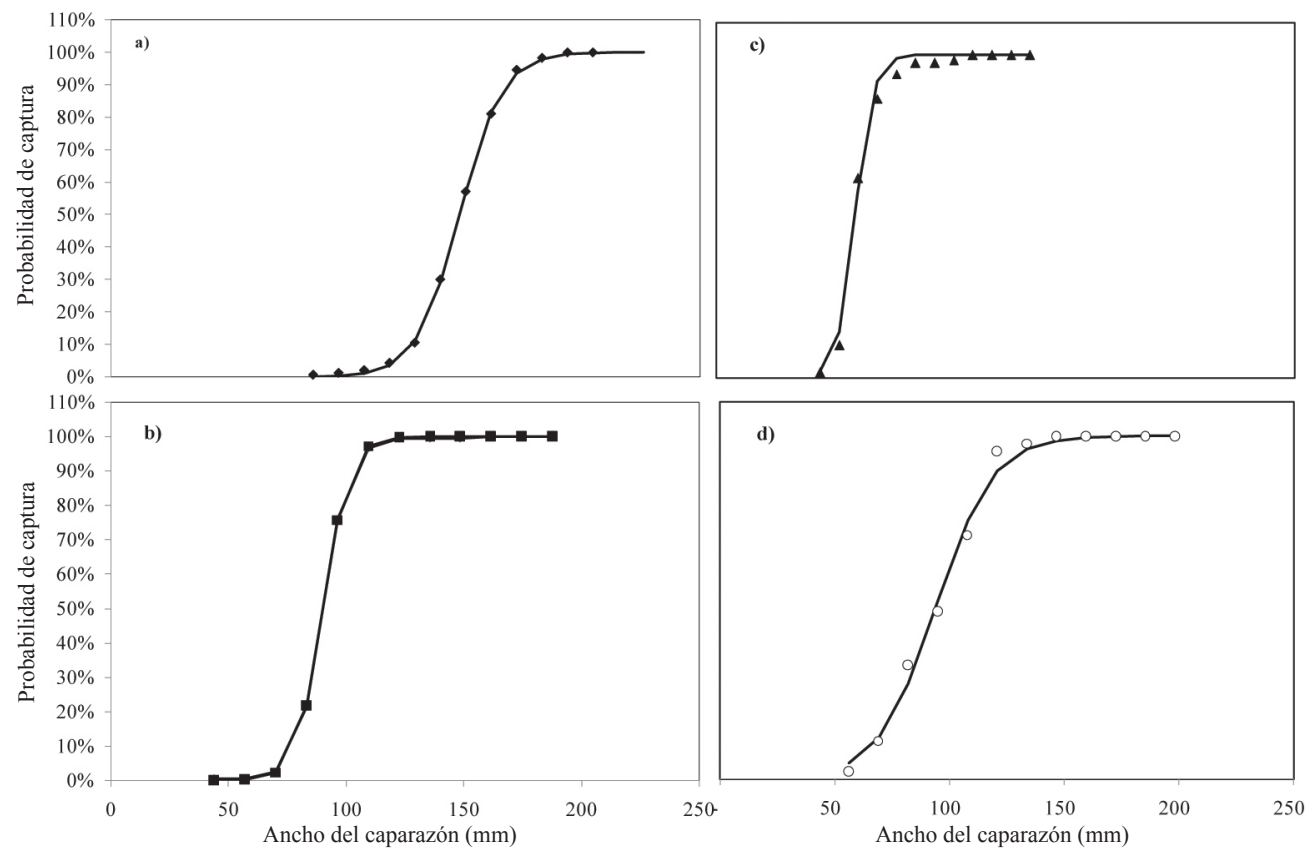

Fig. 5. Curva de selectividad para C. sapidus (a), C. similis (b), L. dubia (c) y $M$. mercenaria (d). Las líneas representan la talla de primera captura $\left(\mathrm{L}_{50}\right)$

Fig. 5. Selectivity curve for C. sapidus (a), C. similis (b), L. dubia (c), and M. mercenaria (d). Lines represent size at first catch $\left(\mathrm{L}_{50}\right)$

cabo un estudio del género Callinectes, reportando una mayor abundancia para C. sapidus $(47.08 \%, \mathrm{n}=121)$ y C. similis $(30.35 \%, \mathrm{n}=78)$. En Venezuela es común encontrar a las especies del género Callinectes como las más abundantes (Moreno et al. 2011). Ahora bien, en la Laguna de Conil, Quintana Roo, se llevó a cabo un estudio en cuanto a la distribución y abundancia de los crustáceos decápodos, donde se reporta que $C$. similis presentó la mayor abundancia con un 55\% de individuos capturados (Magaña-Gallegos et al. 2012).

En la costa de Sisal, Yucatán, la temperatura del agua osciló de 25.0 $\pm 1.06^{\circ} \mathrm{C}$ (época de nortes) a 29.9 $\pm 1.06^{\circ} \mathrm{C}$ (época de lluvias) y una salinidad de $31.11 \pm 1.09$ UPS (época de lluvias) a $33.63 \pm 0.60$ (época de secas). No obstante, Ortiz-León et al. (2007) registraron capturas de jaiba azul a temperaturas de $25.7 \pm 1.9^{\circ} \mathrm{C}$ y salinidades de $8.5 \pm 2.1 \mathrm{ppm}$ en la bahía de Chetumal, Quintana Roo (época de nortes), México. Aunque C. sapidus puede encontrarse en salinidades que van desde 1.9 a 38 UPS (Gómez-Luna et al. 2009). En el caso de L. dubia fue evidente la ausencia de organismos en la época de lluvias y nortes, situación que pudo ser ocasionada por motivos migratorios, ya que en un estudio realizado en New Jersey se ha registrado un descenso en la CPUE en la estación invernal (O’Brien et al. 1999). 
Cuadro 3. Máxima verosimilitud de los parámetros (DE \pm ) de selectividad de cada una de las especies de crustáceos capturadas en la costa de Sisal, Yucatán

Table 3. Maximum likelihood estimates of selectivity parameters ( $\mathrm{SD} \pm$ ) for each crustacean species caught off the coast of Sisal, Yucatan

\begin{tabular}{ccccc}
\hline & C. sapidus & C. similis & L. dubia & M. mercenaria \\
\hline$A$ & $-16.26(0.24)^{*}$ & $-16.7(0.16)^{*}$ & $-15.3968(1.60)^{*}$ & $-7.51(0.55)^{*}$ \\
$B$ & $0.110(0.001)^{*}$ & $0.1853(0.01)^{*}$ & $0.2556(0.02)^{*}$ & $0.08(0.005)^{*}$ \\
$\mathrm{~L}_{50}$ & 147.50 & 90.17 & 60.25 & 93.82 \\
$\mathrm{~L}_{25}$ & 137 & 84.24 & 55.95 & 80.1 \\
$\mathrm{~L}_{75}$ & 157.88 & 96.09 & 64.55 & 107.53 \\
$\mathrm{RS}$ & 19.97 & 11.85 & 8.59 & 27.42 \\
$\mathrm{FS}$ & 11.38 & 6.94 & 4.63 & 7.22 \\
\hline
\end{tabular}

$\mathrm{RS}$, rango de selección; FS, factor de selección; $\mathrm{L}_{25}, \mathrm{~L}_{50} \mathrm{y} \mathrm{L}_{75}$, el ancho del caparazón al cual el $25 \%, 50 \%$ y el $75 \%$ de las diferentes especies de crustáceos capturadas son retenidas en las trampas. $* P<0.001$

$\mathrm{RS}$, selection range; FS, selection factor; $\mathrm{L}_{25}, \mathrm{~L}_{50}$, and $\mathrm{L}_{75}$, carapace width at which $25 \%$, $50 \%$, and $75 \%$ of the different crustacean species caught are retained in the traps. $* P<0.001$

En la figura 4 se observa que los transectos I y III presentaron la menor CPUE, por el contrario, los transectos II y IV presentaron la mayor CPUE. L. dubia registró su máxima CPUE en el transecto II y $M$. mercenaria en el transecto III. La CPUE de $M$. mercenaria y L. dubia podría estar relacionada con las características del hábitat que prefieren estas especies, que son zonas de sedimentación en combinación con las arenoso-fangosas (Cervantes-Martínez \& RamírezGonzález, 2001; Magaña-Gallegos et al. 2012), característico de lagunas costeras, a diferencia del presente estudio que se realizó en la zona costera. Además de que los patrones de movimientos de ciertas especies están relacionados con la búsqueda de un sustrato apropiado para su protección, debido a la depredación, principalmente por peces y moluscos
(López-Abellán et al. 2002). Por otra parte, Ortiz-León et al. (2007) señalan que los factores biológicos, así como los factores físicos, influyen en la distribución y abundancia de las jaibas. La abundancia en la zona de estudio coincide con el trabajo de Gómez-Luna et al. (2009), donde reportan en Santiago de Cuba que C. similis es la segunda especie más abundante, después de C. sapidus. Rocha-Ramírez et al. (1992) refirieron un patrón de abundancia similar.

La proporción sexual de tres de las cuatro especies estudiadas mostró una mayor proporción de hembras en relación con los machos, con excepción de $C$. similis que presentó una relación de 1:1. Lo anterior difiere de lo observado por Ortiz-León et al. (2007), quienes realizaron un estudio de la jaiba azul (C. sapidus) en la bahía de Chetumal, Quintana Roo, donde reportan que por 
cada hembra existen 15 machos. Por otra parte, Gómez-Luna et al. (2009) en un estudio realizado en Cuba, encontraron que $C$. similis exhibió una proporción de 1:3.5 H:M y C. sapidus presentó una proporción de 1:0.40. No obstante, estos últimos estudios se realizaron en hábitats diferentes a los del presente trabajo, ya que el hábitat es un factor importante que está relacionado con la disponibilidad de refugio y alimento, y afecta la distribución de los organismos y la proporción sexual (Andrade-Hernández, 1999; RosasCorrea \& de Jesús-Navarrete, 2008). Otro punto importante es que se le puede atribuir a una mortalidad selectiva, lo que hace que un sexo se vuelva más abundante que otro, o bien, a que puede existir un mecanismo genético por el cual se produzca más de un sexo que el otro, como se ha observado en especies colonizadoras que tienen que maximizar su potencial reproductivo (Estrada, 1999).

La relación ancho del caparazónpeso reveló una tendencia de crecimiento de tipo alométrico para $C$. sapidus, $C$. similis y $L$. dubia, aunque se presentaron diferencias entre sexos, mientras que $M$. mercenaria presentó un crecimiento de tipo isométrico. Es importante mencionar que nuestros resultados coinciden con estudios realizados con especies del género Callinectes. Domínguez et al (1999) y Amador del Ángel et al. (2003) reportan que la especie C. sapidus presenta un tipo de crecimiento alométrico. En los braquiuros parece ser una generalidad asociada con el proceso reproductivo, debido a que las hembras detienen su crecimiento para destinar una gran parte de la energía a la reproducción, en- tretanto los machos continúan creciendo aún después de alcanzar la madurez sexual y la cópula se realiza cuando la hembra está mudada y mientras regenera el exoesqueleto, el macho que la toma debe tener mayor tamaño para protegerla de depredadores (Sastry, 1983).

Las tallas de primera captura $\left(\mathrm{L}_{50}\right)$ de las especies explotadas pueden servir como punto de referencia para la ordenación pesquera y asegurar que los recursos pesqueros se hayan reproducido al menos una vez en su ciclo de vida, antes de ser capturados (Arellano-Torres et al. 2006). La talla de primera captura $\left(\mathrm{L}_{50}\right)$ de la jaiba azul (C. sapidus) del presente estudio (Fig. 5a) se encuentra por arriba de la talla de primera madurez sexual reportada por Andrade-Hernández (1999) en Celestún, Yucatán (población costera más cercana), con 131.8 $\mathrm{mm}$ de AC para las hembras, y $94 \mathrm{~mm}$ de AC para los machos. En el Golfo de México, la talla mínima de captura oficial para C. sapidus es de $110 \mathrm{~mm}$ de AC (DOF, 1974). No obstante, en el presente estudio no se estableció una talla de primera captura por sexo, además de que la captura acumulada al $25 \%\left(\mathrm{~L}_{25}\right)$ estableció una talla de $38 \mathrm{~mm}$ de AC, talla por debajo de la norma legal. Para el género Menippe, Gerhart \& Bert (2008) establecieron una talla de primera madurez sexual de $60 \mathrm{~mm}$ de AC para las hembras y $70 \mathrm{~mm}$ de AC para los machos (híbridos de $M$. mercenaria y $M$. adina), en la Florida, EE.UU. En tanto, para L. dubia se ha reportado una talla de primera madurez sexual de $50.1 \mathrm{~mm}$ 
de AC, en la costa de Yucatán (Espinoza et al. 2003).

\section{CONCLUSIÓN}

De acuerdo con los resultados obtenidos, Callinectes similis, Menippe mercenaria y Libinia dubia son las principales especies de crustáceos que se encuentran asociadas a la captura de jaiba azul (Callinectes sapidus) en Sisal, Yucatán. En la época de secas, C. sapidus y L. dubia registraron su máxima CPUE, sin embargo, $M$. mercenaria y $C$. similis la exhibieron en la época de nortes. La CPUE no presentó variaciones con respecto a los transectos y la profundidad, pero sí entre las especies. C. sapidus registró una mayor abundancia en los cuatro transectos con respecto a la CPUE, mientras que $M$. mercenaria fue la especie que menor abundancia presentó.

Se observó una mayor abundancia de hembras sobre los machos en las especies: C. sapidus, L. dubia y $M$. mercenaria, excepto para C. similis. De forma general, $C$. sapidus, $C$. similis y L. dubia presentaron un crecimiento alométrico, a diferencia de $M$. mercenaria que registró un crecimiento isométrico. De acuerdo con la talla de primera captura $\left(\mathrm{L}_{50}\right)$ quedó establecida de la siguiente manera: C. sapidus registró una talla de $148 \mathrm{~mm}, 90 \mathrm{~mm}$ para C. similis, $60 \mathrm{~mm}$ para L. dubia y, finalmente, $94 \mathrm{~mm}$ para $M$. mercenaria.

\section{AGRADECIMIENTOS}

Al pescador Jesús (don Chucho), Karina Monsreal Vela, Mabel Aguirre
Quintoya los estudiantesqueparticiparon en el proyecto "Estimación poblacional y potencial pesquero de la jaiba azul en Sisal, Yucatán". Asimismo, a Roberto Carlos Barrientos Medina por su valiosa colaboración, y a los tres revisores anónimos, quienes con sus valiosos comentarios contribuyeron a mejorar el contenido de este manuscrito. Gracias a todos por su valiosa colaboración en el trabajo de campo.

\section{BIBLIOGRAFÍA}

Ahumada, M. \& Arana, P. (2009). Pesca artesanal de cangrejo dorado (Chaceon chilensis) en el archipiélago de Juan Fernández, Chile. Lat. Am. J. Aquat. Res., 37(3), 285-296. DOI: http://dx.doi. org/10.3856/vol37-issue3-fulltex-1

Amador del Ángel, L. E., Tejero, G., Damián, I., Miss, F., Cabrera-Rodríguez, P. \& Rivera-Molina, A. (2003, septiembre). Algunos aspectos poblacionales de la jaiba azul (Callinectes sapidus Rathbun 1896) en un centro productor de jaiba suave en la Isla del Carmen. Ponencia presentada en el II Congreso Iberoamericano Virtual de Acuicultura (CIVA) de Campeche, México.

Andrade-Hernández, M. (1999). Pautas para el manejo y ordenamiento de una pesquería multiespecifica de crustáceos en un área natural protegida: El caso de la Ría Celestún, Yucatán. México, México D.F.: Pronatura Península de Yucatán AC.

Arellano-Torres, A., Pérez-Castañeda, R. \& Defeo, O. (2006). Effects of fishing gear on artisanal multiespecific penaeid fishery in a coastal lagoon of Mexico: mesh size, selectivity and management implications. Fish. Manag.Ecol., 
13(5), 309-317. DOI: http://dx.doi. org/10.1111/j.1365-2400.2006.00507.x

Cervantes-Martínez, A. \& Ramírez-González, A. (2001). Abundancia y tallas de Menippe mercenaria (Crustacea: Brachyura), en refugios artificiales en Quintana Roo, México. Rev. Biol. Trop., 49(3-4), 883-888.

DOF. Diario Oficial de la Federación. (1974). Acuerdo por el que se fija la talla mínima para la captura de jaiba en el Golfo de México. México, México D.F.: Sista.

Domínguez, T. L., Rivera, V. R. \& Rodríguez, J. L. S. (1999). Plan de manejo de la pesquería de jaiba en el complejo lagunar Términos Carmen. Campeche, México: Instituto Nacional de la Pesca CRIP-Carmen.

Espinoza, M. J. C., Cervera, C. K., Mena, G. J. C. \& Cob, P. E. F. (2003). Pesca exploratoria con trampas cangrejeras en aguas someras de la costa de Yucatán, México. En B. Espino, M. A. Carrasco, E. G. Cabral \& M. Puente (Eds.), Memorias del II Foro Científico de Pesca Ribereña (pp. 19-20). Colima, México: SAGARPA, INP, CRIP-Manzanillo.

Estrada, V.A.(1999). Aspectos poblacionales de la jaiba Callinectes arcuatus Ordway 1863, en la laguna de Cuyutlán, Colima, México. Tesis de maestría no publicada, Universidad de Colima.

Fitz, H. C. \& Wiegert, R. G. (1992). Local population dynamics of estuarine blue crabs: abundance, recruitment and loss. Mar. Ecol. Prog. Ser., 87, 23-40. DOI: http://dx.doi.org/10.3354/meps087023

Gavaldón, A. \& Fraga, J. (2004, octubre). Nuevos esquemas de uso y acceso a recursos marinos: el enfoque de género para la integración del trabajo $y$ ecosistemas. Ponencia presentada en la Primera Conferencia de Pesquerías Costeras en América Latina y el
Caribe: Evaluando, Manejando y Balanceando Acciones, Mérida, México.

Gerhart, S. D. \& Bert, T. M. (2008). Life-history aspects of stone crab (Genus Menippe): size at maturity, growth and age. J. Crustacean Biol., 28(2), 252-261. DOI: http://dx.doi. org/10.1163/20021975-99990372

Gómez-Luna, L. L., Sosa, M. A., Moreno, C. I. \& Jover, C. A. (2009). Biodiversidad, morfometría y alimentación de los cangrejos del género Callinectes (Decapoda: Portunidae) en Santiago de Cuba. Rev. Biol. Trop., 57(3), 671-686.

INEGI. Instituto Nacional de Estadística y Geografía. (2010). Censo de población $y$ vivienda. Perspectiva Estadística de Yucatán, México: INEGI.

López-Abellán, L. J., Balguerías, E. \& Fernández-Vergaz, V. (2002). Life history characteristics of the deep sea crab Chaceon affinis population off Tenerife (Canary Islands). Fish. Res., 58, 231239. DOI: http://dx.doi.org/10.1016/ S0165-7836(01)00384-8

Magaña-Gallegos, E., Chan-Vivas, E. Y., Poot-López, G. R. \& Reyes-Mendoza, O. F. (2012, octubre). Estudio preliminar de la distribución y abundancia de crustáceos decápodos de interés comercial de la laguna Conil (Yum-Balam), Quintana Roo. Ponencia presentada en la conferencia 64th Gulf and Caribbean Fisheries Institute en Puerto Morelos, México.

Mexicano-Cíntora, C. G., Leonce, V. C. O., Salas, S. \& Vega, C. M. E. (2007). Recursos pesqueros de Yucatán: Fichas técnicas y referencias bibliográficas. Centro de Investigación y Estudios Avanzados del I.P.N. Mérida, México: Academic Edition.

Millar, R. B. \& Fryer, R. J. (1999). Estimating the size-selection curves of 
towed gears, traps, nets and hooks. Rev. FishBiol.Fisher, 9, 89-116.DOI:http:// dx.doi.org/10.1023/A:1008861700420 Moreno, C., Graziani, C., Núñez, J. G. \& Villaroel, E. (2011). Caracterización bioecológica y poblacional de tres comunidades de crustáceos decápodos en la costa noroccidental del Estado Sucre, Venezuela. Zootecnia Trop., 29(1), 29-47.

O’Brien, S. B., Landau, M. \& Able, K. W. (1999). Sex ratios of two species of spider crabs, Libinia dubia H. Milne Edwards, 1834 and L. emarginata Leach, 1815, in the area of Great Bay, New Jersey. Crustaceana, 72(2), 187-192. DOI: http:// dx.doi.org/10.1163/156854099503276

Ortiz-León, H. J., de Jesús-Navarrete, A. \& Sosa, C. E. (2007). Distribución espacial y temporal del cangrejo Callinectes sapidus (Decapoda: Portunidae) en la bahía de Chetumal, Quintana Roo, México. Rev. Biol. Trop., 55(1), 235-245.

Perry, R. I., Walters, C. J. \& Boutillier, J. A. (1999). A framework for providing scientific advice for the management of new and developing invertebrate fisheries. Rev. Fish. Biol. Fisher., 9, 125-150. DOI: http://dx.doi. org/10.1023/A:1008946522213

Raz-Guzmán, A., Sánchez, A. \& Soto, L. (1992). Catálogo ilustrado de cangrejos Braquiuros y Anomuros (Crustacea) de laguna de Alvarado, Veracruz. México. México, México D.F. Instituto de Biología, UNAM: Editorial Universitaria. Rocha-Ramírez, A., Cházaro-Olvera, S. \& Mueller-Meier, P. M. (1992). Ecología del género Callinectes (Brachyura: Portunidae) en seis cuerpos de agua costeros del estado de Veracruz, México. An. Inst. Cien. del Mar y Limnol. UNAM, 19, 33-41.

Rosas-Correa, C. O. \& de Jesús-Navarrete, A. (2008). Parámetros poblacionales de la jaiba azul Callinectes sapidus (Rathbun, 1896) en la bahía de Chetumal, Quintana Roo, México. Rev. Biol. Mar. Oceanog., 43(2), 247253. DOI: http://dx.doi.org/10.4067/ S0718-19572008000200003

Sakia, R. M. (1992). The Box-Cox transformation technique: a review. The Statistician, 41, 169-178. DOI: http:// dx.doi.org/10.2307/2348250

Sastry, A. N. (1983). Ecological aspects of reproduction. En F. J. Vernberg \& W. B. Vernberg (Ed.), The biology of crustacean (pp. 179-270). New York, EE.UU.: Academic Press.

Smith, M. T. \& Addison, J. T. (2003). Methods for stock assessment of crustacean fisheries. Fish. Res., 65, 231256. DOI: http://dx.doi.org/10.1016/j. fishres.2003.09.017

Tully, O., Freire, J. \& Addison, J. (2003). Crustacean fisheries. Fish. Res., 65, 1-2. DOI: http://dx.doi.org/10.1016/j. fishres.2003.09.003

Uc-Espadas, M. C. (2007). Estrategias de vida en hogares costeros, estudio de caso en Celestún, Yucatán. Tesis de maestría no publicada. Centro de Investigación y de Estudios Avanzados del Instituto Politécnico Nacional. Unidad Mérida.

Vega-Cendejas, M. E. (2004). Ictiofauna de la Reserva de la Biosfera Celestún, Yucatán: una contribución al conocimiento de su biodiversidad. Anales del Instituto de Biología, U.N.A.M., Serie Zoología, 75(1), 193-206.

Velázquez-Abunader, J. I., HernándezHerrera, A., Martínez-Aguilar, S., DíazUribe, J. G. \& Morales-Bojórquez, E. (2012). Interannual variability in mantle length structure, recruitment, and sex ratio of jumbo squid, Dosidicus gigas, in the Central Gulf of California, México. J. Shellfish Res., 31(1), 125-134. DOI: http://dx.doi.org/10.2983/035.031.0116 